Int. J. Odontostomat., 5(3):279-286, 2011.

\title{
Terapia Periodontal Mediante Proteínas Derivadas del Esmalte y Aloinjerto Óseo
}

\author{
Periodontal Therapy by Enamel Matrix-derived Protein and Bone Allograft
}

\author{
Gustavo Alfonso Martínez López;; Lizette Llamosa Cañez; Víctor Javier Beltrán Varas*; \\ Mario Cantín $^{* * *}$ \& Ramón Fuentes Fernández ${ }^{* * *}$
}

\begin{abstract}
MARTÍNEZ, G.; LLAMOSA, L.; BELTRÁN, V.; CANTÍN, M. \& FUENTES, R. Terapia periodontal mediante proteínas derivadas de la matriz del esmalte y aloinjerto óseo. Int. J. Odontostomat., 5(3):279-286, 2011.

RESUMEN: Se describe el tratamiento periodontal en un defecto óseo situado mesial a la pieza 2.3 y distal a la pieza 2.2, mediante la terapia combinada de derivado de la matriz del esmalte (DME) y aloinjerto óseo mineralizado. Se valoró la zona afectada y se planeo su abordaje quirúrgico para la terapia combinada. Seis meses después del tratamiento se observó una reducción significativa en la profundidad al sondeo y ganancia en los niveles de inserción, así como el relleno óseo del defecto en la evaluación radiográfica. Los resultados reafirman la efectividad del DME y el aloinjerto óseo mineralizado en la terapia regenerativa periodontal.
\end{abstract}

PALABRAS CLAVE: proteínas de la matriz del esmalte, aloinjerto óseo, regeneración periodontal.

\section{INTRODUCCIÓN}

La terapia periodontal incluye dentro de sus objetivos principales detener el avance de la enfermedad, prevenir la recurrencia de la enfermedad y regenerar los tejidos periodontales perdidos. Este último objetivo, se define como la reconstrucción de un ligamento periodontal funcionalmente orientado, insertándose en nuevo hueso alveolar y cemento, lo cual únicamente puede ser determinado mediante un examen histológico de la cicatrización de los tejidos después de la cirugía. Se ha presentado evidencia de regeneración periodontal en humanos en distintas revisiones de literatura, siendo esta limitada, ya que requiere de cortes histológicos. Los clínicos deben confiar en parámetros tales como reducción de la profundidad de bolsa, ganancia de niveles de inserción clínica, evidencia radiográfica y reentrada al relleno óseo del defecto, para evaluar clínicamente una modalidad de tratamiento (Froum et al., 2001a).

Hace poco más de una década la terapía periodontal introdujo a las proteínas derivadas de la matriz del esmalte (DME), demostrando potencial para mediar la regeneración periodontal tanto en humanos como en modelos animales. Este material está compuesto primariamente de amelogenina y proteínas que se derivan de los brotes dentales porcinos (Hammarström, 1997), imitando la function de la vaina epitelial radicular.

El uso de DME en el tratamiento de defectos intraóseos ha mostrado resultados significativos en el mejoramiento de la profundidad de bolsa, niveles de inserción clínicos y relleno óseo (Froum et al., 2001b), además de ser un factor crucial en el inicio de la formación del cemento radicular acelular, estimulación del desarrollo del ligamento periodontal y del hueso alveolar (Cattaneo et al., 2003).

Algunos autores consideran esencial el empleo de injertos óseos en la restauración del hueso perdido acompañado por un aparato de inserción funcional (Bowers et al., 1989; Mellonig, 1992). Los objetivos de los injertos óseos son reducción de la bolsa, ganancia en los niveles de inserción clínica, relleno óseo del defecto, regeneración de nuevo hueso, cemento y ligamento periodontal (Schallhorn, 1977; Brunsvold \&

\footnotetext{
* Programa de Magister en Ciencias Odontológicas con Especialidad en Periodoncia, Universidad Autónoma de Nuevo León, México.

* Programa de Magister en Odontología, Universidad de La Frontera, Chile.

${ }^{*}$ Departamento de Odontología Integral, Facultad de Medicina, Universidad de La Frontera, Chile.
} 
Mellonig, 1993). De los diferentes tipos de injertos, los aloinjertos corresponden a biomateriales que son transferidos entre miembros genéticamente diferentes de la misma especie, donde el aloinjerto óseo mineralizado (Puros $®$, Zimmer Dental) ha sido introducido como sustituto óseo. Estudios demuestran que el injerto óseo mineralizado parece ser un material eficaz en el tratamiento de defectos intraóseos (Browning et al., 2009). La combinación de DME ha sido utilizado con otro tipo de injertos (Hoidal et al., 2008), pero no hemos encontrado reportes de la combinación de DME con aloinjerto en la evidencia disponible.

El propósito del presente artículo es evaluar los resultados clínicos del tratamiento de un defecto óseo combinado de 2 y 3 paredes mediante la aplicación de DME y aloinjerto óseo mineralizado en un período de 6 meses.

\section{REPORTE DEL CASO}

Paciente femenino de 58 años de edad, referida por la clínica de Periodoncia pregrado se presenta en el Posgrado de Periodoncia de la Universidad Autónoma de Nuevo León. El motivo de la consulta fue "sangrado de encías e inflamación". Menciona que su último tratamiento dental fue un puente parcial fijo. La paciente refiere encontrarse bajo tratamiento médico para control de la hipertensión y diabetes mediante Metformina 850 mg/día, Losartan 25 mg/día y Aspirina 500 mg/día. La paciente presentaba una presión arterial de 130/85 $\mathrm{mmHg}$. Se clasificó como un paciente ASA II. No se relatan hábitos de consumo de tabaco y alcohol.

En el exámen clínico se observó inflamación gingival generalizada y de mayor tonalidad en el área de la papila mesial en la pieza 2.3. Presencia de irritantes locales generalizados, con mayor presencia en anteroinferiores, higiene bucal y restauraciones protésicas deficientes. El sondeo periodontal completo arrojó profundidades de surco de 1 a $3 \mathrm{~mm}$ generalizado, profundidad de bolsas localizadas de $4 \mathrm{~mm}$ en ambas arcadas. En la zona a tratar se localizó una bolsa de $8 \mathrm{~mm}$ y niveles de inserción de 8 a $9 \mathrm{~mm}$ en la pieza 2.3. Las piezas $2.2,2.3$ y 2.4 presentaban recesiones clase III, basado en la clasificación de Miller. El examen radiográfico reveló zonas con pérdida ósea horizontal leve generalizada, defectoóseo vertical moderado en la pieza 2.3, aparentemente de 2 paredes, con ensanchamiento del ligamento hacia la zona del ápice radicular, sin presencia de movilidad. Se clasificó como un caso
ADA tipo II, Periodontitis crónica leve generalizada, moderada localizada en 2.3 (Armitage, 1999). El pronóstico general fue bueno. El pronóstico individual de cada pieza para el área tratada fue: 2.2 regular, 2.3 regular, 2.4 bueno.

Se presentaron y explicaron a la paciente las distintas opciones de tratamiento, definiendo la terapéutica de la forma siguiente: fase sistémica, interconsulta con su médico, se le indicó a la paciente suspender el antiagregante plaquetario 2 días antes del procedimiento quirúrgico; fase higiénica, educación al paciente respecto a la etiología y prevención de la enfermedad periodontal, instrucción, evaluación y reforzamiento del control de placa, destartraje, curetaje y alisado radicular ambas arcadas.

Posteriormente se deribó a interconsulta con el departamento de Endodoncia para realizar el tratamiento endodóntico de la pieza 2.3 previo al procedimiento quirúrgico, planificado con colgajo por desbridación en las piezas 2.2 a 2.3 con utilización de DME en combinación con injerto óseo mineralizado, así como colgajo posicionado coronal de 2.2 a 2.4 .

Procedimiento quirúrgico. Se realizaron incisiones para preservación de papila gingival utilizando la técnica de Takei, a la cual se le realizó una modificación, esto para obtener un mejor acceso al defecto (Takei et al., 1985). Se realizó el abordaje por palatino, extendiendo las incisiones intrasulculares en dos incisiones verticales ligeramente divergentes desde el ángulo línea mesial y distal de 2.3-2.2 respectivamente, 2 a 3 $\mathrm{mm}$ hacia apical y conectando con una incisión horizontal. Se reflejó la papila hacia vestibular. El colgajo vestibular se diseño de la siguiente manera: se realizó incisión intrasulcular en la pieza 2.4, se continuó diseñando papila quirúrgica, incisiones intrasulculares en 2.3 y 2.2, terminando con una incisión vertical biselada en el ángulo línea mesial de 2.2 y pasando la unión mucogingival. Se reflejó colgajo de espesor total y liberando el colgajo a espesor parcial en su parte apical y se depitelizaron las papilas. Se desbridó, se retiró el cálculo remanente y se alisaron las raíces. Se sondeo el defecto siendo este de aproximadamente $10 \mathrm{~mm}$ desde el fondo del defecto hasta la cresta ósea. Se acondicionaron las raíces con PrefGelTM (EDTA al 24\%) siguiendo las recomendaciones del fabricante. Posteriormente se irrigó abundantemente y se aplicó DME (Emdogain $®)$ sobre la base del defecto y en las raíces de las piezas a tratar, evitando la contaminación con sangre o saliva. Después se colocó el aloinjerto (Puros ${ }^{8}$, Zimmer Dental) y sobre este se aplicó DME (Browning et al.) Se 
posicionó coronalmente el colgajo (Bernimoulin et al., 1975), utilizando catgut 5-0, puntos directos y suturando la base de la papila por palatino con puntos directos (Fig. 1). Por último se aplicó DME sobre los bordes de las incisiones dejándolo por unos minutos. Dentro de los cuidados postoperatorios se instruyó a la paciente en el uso de clorhexidina al $0,12 \% 3$ veces al día durante 4 semanas postoperatorias, además de evitar el cepillado de la zona tratada durante 3 semanas. La paciente recibió tratamiento antibiótico (Amoxicilina 500

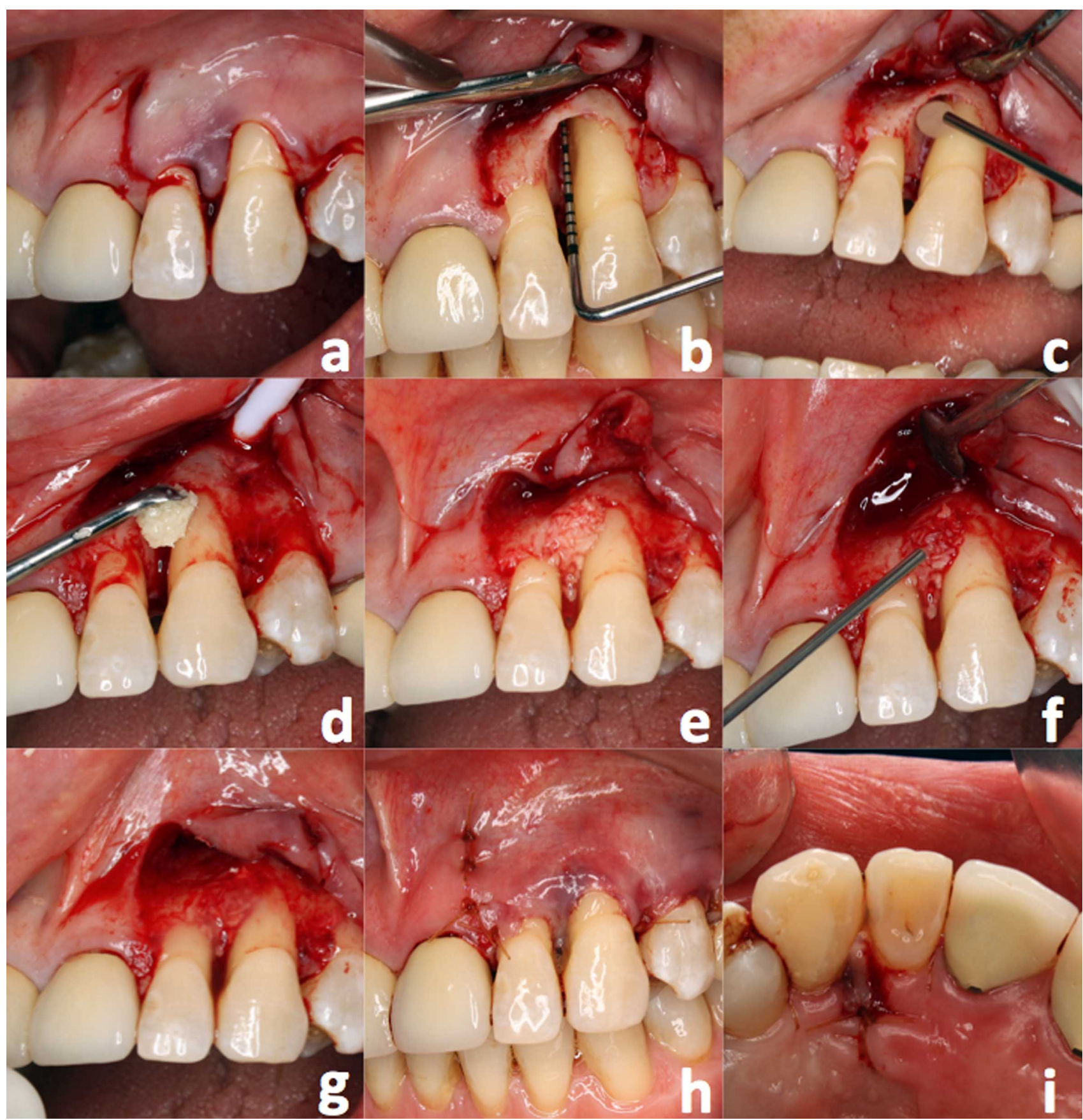

Fig. 1. Secuencia quirúrgica utilizada para realizar la terapia combina de DME y aloinjerto ósea. (a). Colgajo vestibular mediante incisión intrasulcular desde pieza 2.4 diseñando papila quirúrgica, hasta la pieza 2.2, terminando con una incisión vertical biselada en el ángulo línea mesial de 2.2 y pasando la unión mucogingival. (b). Levantamiento del colgajo de espesor total y sondaje del defecto de aproximadamente $10 \mathrm{~mm}$ hasta la cresta ósea. (c). Aplicación de DME (Emdogain®) sobre la base del defecto y en la raíz de las pieza previo acondicionamiento radicular con PrefGelTM. (d y e). Colocación del aloinjerto (Puros ${ }^{\circledR}$, Zimmer Dental). (f y g). Aplicación final de DME sobre injeto óseo modelado. (h). Posicionamiento coronal del colgajo y sutura con puntos directos. (i) Suturan de la base de la papila por palatino con puntos directos. 


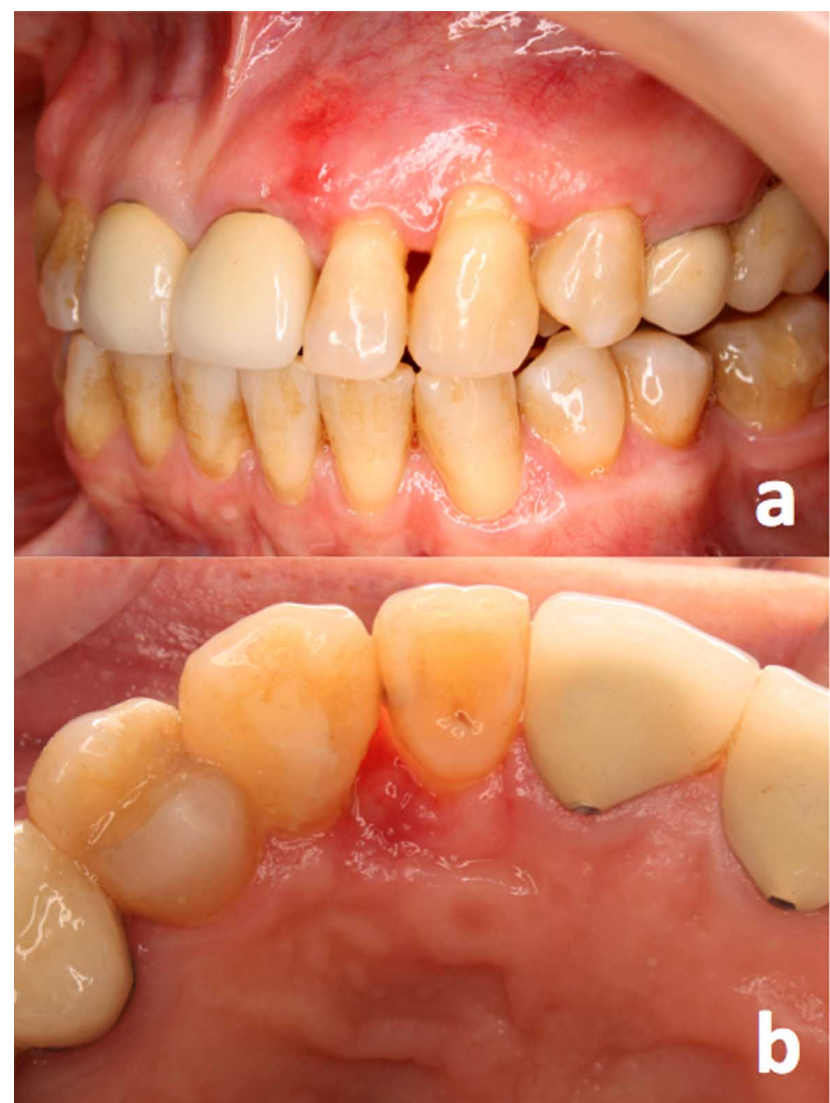

Fig. 2. Control y retiro de suturas a las 2 semanas post operatorias; zona quirúrgica presenta una cicatrización óptima en la zona vestibular (a) y palatina (b).

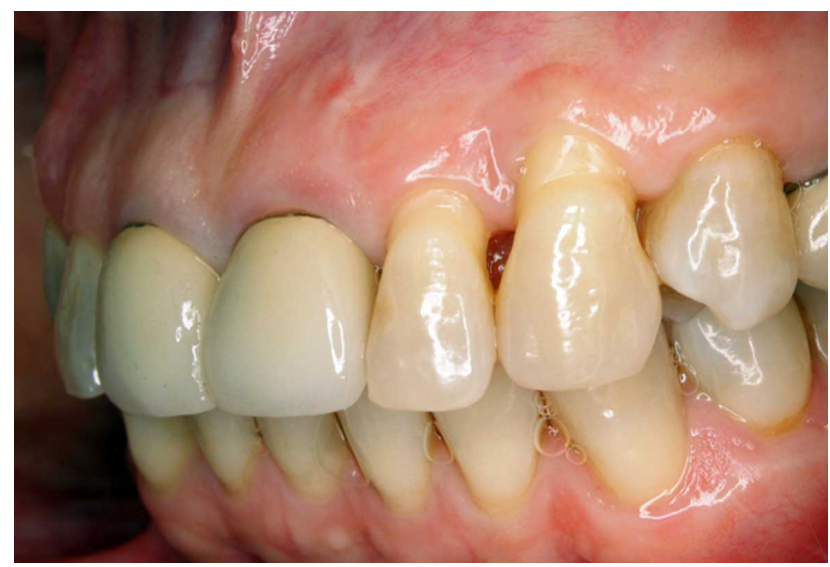

Fig. 3. Control postquirúrgico con una evolución de 6 meses donde se evaluaron los niveles de inserción y profundidad de sondaje

mg, 1 cápsula cada 8 horas durante 7 días) y analgésico/antiinflamatorio (Ibuprofeno $600 \mathrm{mg}, 1$ cápsula cada 8 horas durante 5 días y Dexametazona $4 \mathrm{mg}$ IM, 1 ampolla previo al procedimiento quirúrgico). Se retiraron las suturas a las 2 semanas posteriores (Fig. 2). Se realizó control de placa y pulido de las superfi-

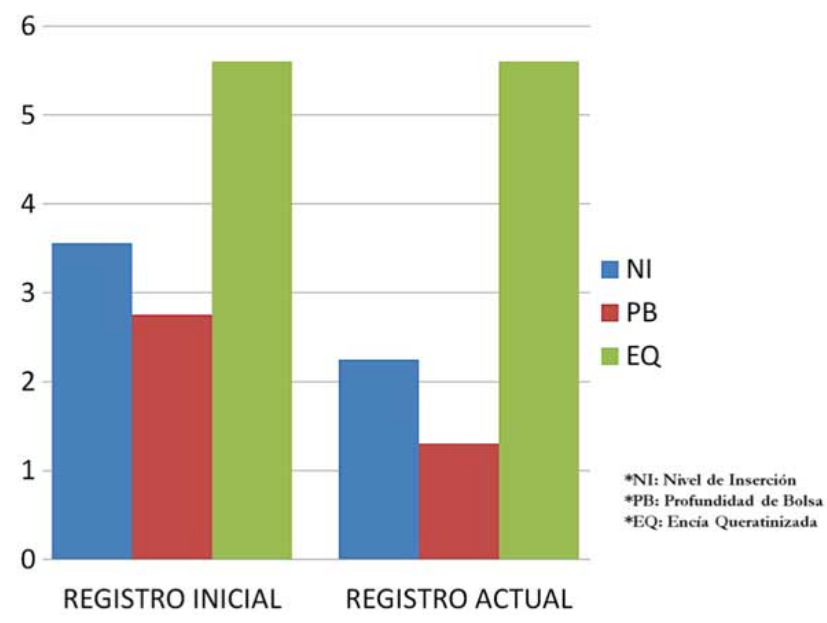

Fig. 4. Evaluación de los niveles de inserción, profundidad de sondeo y cantidad de encía queratinizada iniciales (prequirúrgicos) y actuales (6 meses de evolución postquirúrgica). Se observa gráficamente ganancia en los niveles de inserción clínicos, reducción en la profundidad al sondeo y la mantención de la cantidad de encía queratinizada a los 6 meses.

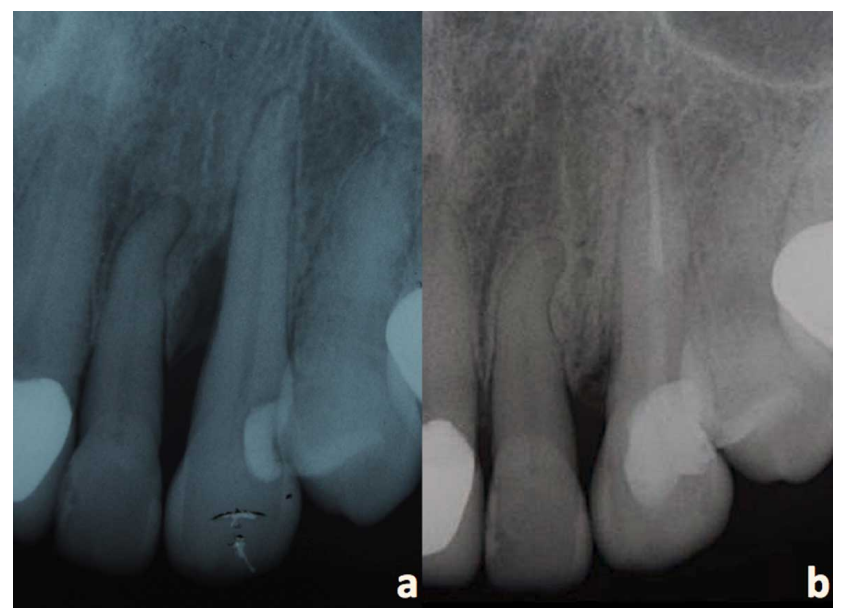

Fig. 5. Examen radiográfico periapical de la pieza 2.3. (a). Radiografía preoperatoria donde se observa el extenso defecto óseo por mesial a la raíz de la pieza 2.3. (b). Radiografía de control postquirúrgico de 6 meses de evolución donde se observa la desaparición del defecto óseo manteniendo sólo una reabsorción ósea marginal. El relleno óseo muestra una adecuada radiolucidez semejante al tejido óseo circundante. No se observa lesión ósea apical posterior al tratamiento endodóntico.

cies dentales durante las primeras cuatro semanas postoperatorias (Mellonig, 1999). Se realizó el control postoperatorio (Fig. 3), y se evaluaron los niveles de inserción, profundidad de sondeo (Fig. 4), junto al control radiográfico postquirúrgicos con una evolución de 6 meses (Fig. 5). 


\section{DISCUSIÓN}

La regeneración periodontal mediante el uso de DME por sí sola ha sido bien documentada en estudios clínicos y reportes de caso. Tonneti et al. (2002) realizaron un estudio multicéntrico controlado al azar observando que el uso de DME en combinación con la preservación de papila gingival proveen un beneficio adicional en el tratamiento regenerativo de defectos intraóseos, con una mejoría en los niveles de inserción clínicos de $0,5 \pm 0,2 \mathrm{~mm}$ y una significativa reducción de la profundidad de sondeo, sin diferencias en cuanto a la recesión gingival entre el grupo evaluado y grupo control. Meyle et al. (2004) en estudio multicentrico al azar compararon el tratamiento regenerativo mediante DME y con regeneración tiular guiada (RTG) usando membranas bioabsorbibles. Los resultados fueron similares entre ambos procedimientos, pero encontraron un menor nivel de recesión gingival en los sitios tratados con DME. Por otra parte, encontraron que los sitios tratados con membranas presentaron un mayor número de cambios negativos en cuanto al nivel óseo, mientras los sitios tratados con DME no tuvieron cambios. Esto significa que no ocurrió reabsorción ósea en los sitios tratados con DME mientras hubo una ligera reabsorción usando membranas.

La información disponible acerca del injerto óseo mineralizado en el tratamiento de defectos periodontales es limitada. Browning et al. evaluaron la eficacia del aloinjerto óseo mineralizado (Puros) en el cual obtuvieron una mayor ganancia de relleno óseo, $4,1 \mathrm{~mm}-67 \%$, en comparación con estudios en los que se usó DFDBA, observándose $1,7 \mathrm{~mm}-59 \%$ (Rummelhart, 1984), 2,6 mm - 52\% (Mellonig, 1984), 2,2 mm - 61\% (Bowers et al.), 1,9 mm - 58\% (Guillemin et al., 1993) y $2,2 \mathrm{~mm}-52 \%$ (Masters et al., 1996).

Efectos clínicos beneficiosos se han demostrado con la adición de DME al tratamiento con aloinjertos de hueso desmineralizado liofilizado en comparación con DME solo (Gurinsky et al., 2004), mientras que al evaluar la eficacia de DFDBA combinado con DME, en comparación con DFDBA solo en el tratamiento de defectos intraóseos en casos de periodontitis crónica, ambos tratamientos se muestran seguros y eficaces para los defectos periodontales, sin embargo, la adición de DME para DFDBA proporcionan una mejoría estadísticamente significativa de los parámetros de medición en los tejidos blandos y duros (Hoidal et al., 2008).
El área tratada quirúrgicamente mediante $\mathrm{DME}$ y aloinjerto óseo mineralizado (Puros $®$ ) del presente reporte mostró una ganancia en los niveles de inserción clínicos (Â=1,31 mm) así como reducción en la profundidad al sondeo $(\hat{A}=1,45 \mathrm{~mm})$, logrando mantener la cantidad de encía queratinizada $(\hat{A}=5,6 \mathrm{~mm})$ a los 6 meses (Figura 5). Estos resultados concuerdan con los descrito por Saito et al. (2010), quienes realizaron un estudio clínico retrospectivo evaluando el tratamiento quirúrgico de defectos periodontales intaóseos con DME durante las primeras etapas de cicatrización hasta los 6 meses, observando una mejora significativa en profundidad al sondaje y ganancia del nivel de inserción a los 3 meses, siendo significativa a los 6 meses (mejora del 59\%). Kalpidis \& Ruben (2010) mediante un meta-análisis de estudios clínicos sobre el manejo de defectos óseos angulares con DME reveló una reducción de profundidad al sondaje de $4 \mathrm{~mm}$ ( $50 \%$ de la profundidad al sondaje base) y una ganancia del nivel de inserción clínica de 3,2mm (33\% del nivel de inserción original) para un total de 317 lesiones durante un período de observación de 6 a 12 meses. Los resultados del presente reporte son comparable a esos resultados en términos de eficacia de la terapia con DME.

Radiográficamente se observó buen relleno óseo del defecto a los 6 meses de evolución (Fig. 4). Esto concuerda con lo expresado por Saito et al. quienes mencionan que se puede encontrar una leve mejora en la radioopacidad de las lesiones en los primeros 3 meses postoperatorios, pero signos de posible relleno óseo son significativamente aparentes a los 6 meses, sugirieron que el tratamiento de defectos óseos mediante la terapia de DME combinada con aloinjertos induce una cicatrización ósea favorable con un alto nivel de predictibilidad. Este adecuado relleno óseo, teóricamente es en mayor medida derivado del aloinjerto que del DME, pero resulta muy interesante la combinación con la DME, ya que la DME sugiere posiblemente un efecto inhibitorio sobre las células epiteliales, por tanto también de la epitelización durante las etapas de regeneración temprana (Kalpidis \& Ruben), siendo un efecto deseable en cualquier terapia periodontal de regeneración ósea.

Nuestras observaciones clínicas concuerdan con las investigaciones realizadas por Prata et al. (2007), quienes evaluaron histológica e histome- 
tricamente la reparación ósea en los defectos intraóseos después de extracciones dentales en ratas con una combinación de DME e injerto de hueso autógeno, donde la cuantificación de la reparación ósea en las zonas adyacentes al injerto mostró que el hueso autógeno asociados a DME produce una mayor cantidad de hueso. Se concluyó que el hueso autógeno asociados a DME injertado en defectos óseos, inmediatamente después de la extracción dental en ratas, demostró biocompatibilidad y aceleración de la reparación del defecto óseo. Recientemente Joing et al. (2011) investigaron la influencia de las DME sobre la proliferación, diferenciación y adhesión de los osteoblastos de hueso alveolar humanos in vitro, encontrando que las DME aumenta significativamente el crecimiento de la población de osteoblastos de hueso alveolar humanos, pero disminuye su diferenciación osteoblástica y adhesión. Efectos inhibitorios sugieren que las DME pueden permitir que otros tipos de células regeneren el tejido periodontal al inhibir la diferenciación osteoblástica y la adhesión, que en parte podría explicar la formación del aparato periodontal y no una simple anquilosis después de la aplicación de DME y aloinjertos sobre defectos óseos periodontales.

Debemos mencionar que en nuestro caso el diente tratado no presentaba movilidad en la evaluación inicial, y no se reportaron cambios en todo el periodo post operatorio, siendo este un importante factor de predicción de éxito, ya que se ha sugerido que la movilidad dentaria es un factor de riesgo para la futura pérdida de inserción cuando se compara el tratamiento de defectos óseos con DME en dientes con y sin movilidadad (Saito et al.).

Resulta interesante evaluar el conjunto de mejorías en el estado de los tejidos blandos observadas en el periodo de evaluación. Autores como AlHezaimi et al. (2011) evaluaron histométricamente el cambio en el grosor del tejido blando gingival alrededor de los defectos tipo dehiscencia tratados con DME en perros Beagle comparándolos con un grupo control. Los análisis histométricos mostraron que el espesor medio del tejido gingival fue de 1,76 $\pm 0,23 \mathrm{~mm}$ en los sitios tratados con DME en comparación con $0,72 \pm 0,11 \mathrm{~mm}$ para los sitios de control. Parece que el DME tiene una influencia positiva en el aumento de grosor del tejido gingival. Estos resultados proponen una nueva justificación para el aumento de la previsibilidad de los colgajos de avance coronario en lograr una cobertura completa de la raíz cuando se usa conjuntamente DME. Con- trario a estas observaciones, Del Pizzo et al. (2005) al evaluar la capacidad del DME para mejorar la cobertura de la raíz utilizando la técnica quirúrgica de colgajo de avance coronal durante 2 años de seguimiento en lesiones Miller Clase II, sugirien que el uso adicional de DME al colgajo de avance coronal no se justifica cuando se busca solamente mejorar la cobertura de la raíz; sin embargo la combinación de estos si es efectiva cuando se busca lograr la regeneración periodontal en lugar de la simple reparación. Estas observaciones podrían explicar nuestras resultados clínicos, en donde se combinó la técnica quirúrgica con la DME y el aloinjerto, logrando la regeneración del conjunto de tejidos periodontales, donde el punto más crítico será el manejo del periodoncista al realizar una adecuada conservación de los tejidos interdentales y la adaptación óptima del tejido blando por cierre primario esesencial para obtener buenos resultados, tal como es descrito en esta experiencia mediante el cuidado de las papilas gingivales y la utilización de un colgajo de posicionamiento coronal.

Finalmente, la terapéutica combinada utilizada también es ventajosa en términos de costo-efectividad. List et al. (2010) identificaron el mejor enfoque costo-efectividad para el tratamiento de lesiones infraóseas, donde el tratamiento con DME es costo-efectivo (1 año de perspectiva). Sin embargo, el mejor costo-beneficio/efectividad fue alcanzado por el tratamiento con DME en conjunto con vidrio bioactivo o injerto óseo, mientras que la aplicación adicional de plasma rico en plaquetas (PRP) o una membrana reabsorbible generó un costo extremadamente alto, concluyendo que si el uso de DME está indicado, éste junto con vidrio bioactivo o sustitutos óseos son más rentable que el DME solo a largo plazo, y el uso adicional de PRP o membranas sólo puede justificarse cuando los recursos monetarios para el tratamiento estén al alcance, y su utilización sea justificada.

En conclusión, después de 6 meses de evolución posterior al tratamiento del defecto óseo mencionado con DME y aloinjerto óseo mineralizado, se observó clínicamente una mejoría en el estado de los tejidos blandos, buen nivel del llenado óseo del defecto en la evaluación radiográfica y ganancia en los niveles de inserción clínicos así como reducción de la profundidad al sondeo. Los resultados obtenidos mediante este tratamiento reafirman y recomiendan la efectividad del DME y el aloinjerto óseo mineralizado en la terapia regenerativa. 
MARTÍNEZ, G.; LLAMOSA, L.; BELTRÁN, V.; CANTÍN, M. \& FUENTES, R. Periodontal therapy by enamel matrix-derived protein and bone allograft.Int. J. Odontostomat., 5(3):279-286, 2011.

ABSTRACT: We describe the periodontal treatment in a bone defect located mesial to the 2.3 tooth, using combination therapy of enamel matrix derivate (EMD) and mineralized bone allograft. We evaluated the affected area and planned their surgical approach to combination therapy. Six months after treatment it showed a significant reduction in probing depth and gain in attachment levels and bone filling of the defect in the radiographic evaluation. These results confirm the effectiveness of the EMD and the mineralized bone allograft in periodontal regenerative therapy.

KEY WORDS: proteins of enamel matrix, allograft bone, periodontal regeneration.

\section{REFERENCIAS BIBLIOGRAFICAS}

Al-Hezaimi, K.; Al-Fahad, H.; O'Neill, R.; Shuman, L. \& Griffin, $\mathrm{T}$. The effect of enamel matrix protein on gingival tissue thickness in vivo. Odontology, 2011. DOI 10.1007/ s10266-011-0022-5

Armitage, G. C. Development of a classification system for periodontal diseases and conditions. Ann. Periodontol., 4:1-6, 1999.

Bernimoulin, J. P.; Lüscher, B. \& Mühlemann, H. R. Coronally repositioned periodontal flap. Clinical evaluation after one year. J. Clin. Periodontol., 2:1-13, 1975.

Bowers, G. M.; Chadroff, B.; Carnevale, R.; Mellonig, J.; Corio, R.; Emerson, J.; Stevens, M. \& Romberg, E. Histologic evaluation of new attachment apparatus formation in humans. Part III. J. Periodontol., 60:683-93, 1989.

Browning, E. S.; Mealey, B. L. \& Mellonig, J. T. Evaluation of a mineralized cancellous bone allograft for the treatment of periodontal osseous defects: 6-month surgical reentry. Int. J. Periodontics Restorative Dent., 29:41-7, 2009.

Brunsvold, M. A. \& Mellonig, J. T. Bone grafts and periodontal regeneration. Periodontol. 2000, 1:80-91, 1993.

Cattaneo, V.; Rota, C.; Silvestri, M.; Piacentini, C.; Forlino, A.; Gallanti, A.; Rasperini, G. \& Cetta, G. Effect of enamel matrix derivative on human periodontal fibroblasts: proliferation, morphology and root surface colonization. An in vitro study. J. Periodontal Res., 38:568-74, 2003.

Del Pizzo, M.; Zucchelli, G.; Modica, F.; Villa, R. \& Debernardi, C. Coronally advanced flap with or without enamel matrix derivative for root coverage: a 2-year study. J. Clin. Periodontol., 32:1181-7, 2005.

Froum, S. J.; Weinberg, M. A.; Rosenberg, E. \& Tarnow, D. A comparative study utilizing open flap debridement with and without enamel matrix derivative in the treatment of periodontal intrabony defects: a 12-month re-entry study. J. Periodontol., 72:25-34, 2001a.
Froum, S.; Lemler, J.; Horowitz, R. \& Davidson, B. The use of enamel matrix derivative in the treatment of periodontal osseous defects: a clinical decision tree based on biologic principles of regeneration. Int. J. Periodontics Restorative Dent., 21:437-49, 2001b.

Guillemin, M. R.; Mellonig, J. T. \& Brunsvold, M. A. Healing in periodontal defects treated by decalcified freeze-dried bone allografts in combination with ePTFE membranes (I). Clinical and scanning electron microscope analysis. J. Clin. Periodontol., 20:528-36, 1993.

Gurinsky, B. S.; Mills, M. P. \& Mellonig, J. T. Clinical evaluation of demineralized freeze-dried bone allograft and enamel matrix derivative versus enamel matrix derivative alone for the treatment of periodontal osseous defects in humans. J. Periodontol., 75:1309-18, 2004.

Hammarström, L. Enamel matrix, cementum development and regeneration. J. Clin. Periodontol., 24:658-68, 1997.

Hoidal, M. J.; Grimard, B. A.; Mills, M. P.; Schoolfield, J. D.; Mellonig, J. T. \& Mealey, B. L. Clinical evaluation of demineralized freeze-dried bone allograft with and without enamel matrix derivative for the treatment of periodontal osseous defects in humans. J. Periodontol., 79:2273-80, 2008.

Jiang, S. Y.; Shu, R.; Song, Z. C. \& Xie, Y. F. Effects of enamel matrix proteins on proliferation, differentiation and attachment of human alveolar osteoblasts. Cell Prolif., 44:372-9, 2011.

Kalpidis, C. D. \& Ruben, M. P. Treatment of intrabony periodontal defects with enamel matrix derivative. $J$. Periodontol., 73:1360-76, 2002.

Listl, S.; Tu, Y. K. \& Faggion, C. M. Jr. A cost-effectiveness evaluation of enamel matrix derivatives alone or in conjunction with regenerative devices in the treatment of periodontal intra-osseous defects. J. Clin. Periodontol., $37: 920-7,2010$ 
MARTíNEZ, G.; LLAMOSA, L.; BELTRÁN, V.; CANTíN, M. \& FUENTES, R. Terapia periodontal mediante proteínas derivadas de la matriz del esmalte y aloinjerto óseo. Int. J. Odontostomat., 5(3):279-286, 2011.

Masters, L. B.; Mellonig, J. T.; Brunsvold, M. A. \& Nummikoski, P. V. A clinical evaluation of demineralized freeze-dried bone allograft in combination with tetracycline in the treatment of periodontal osseous defects. J. Periodontol., 67:770-81, 1996.

Mellonig, J. T. Decalcified freeze-dried bone allograft as an implant material in human periodontal defects. Int. J. Periodontics Restorative Dent., 4:40-55, 1984.

Mellonig, J. T. Autogenous and allogeneic bone grafts in periodontal therapy. Crit. Rev. Oral Biol. Med., 3:333-52, 1992.

Mellonig, J. Periodontal reconstructive surgery: technique and clinical and histological case report. Int. J. Periodontics Restorative Dent., 19:9-19, 1999.

Meyle, J.; Gonzales, J. R.; Bödeker, R. H.; Hoffmann, T.; Richter, S.; Heinz, B.; Arjomand, M.; Reich, E.; Sculean, A.; Jepsen, K. \& Jepsen, S. A randomized clinical trial comparing enamel matrix derivative and membrane treatment of buccal class II furcation involvement in mandibular molars. Part II: secondary outcomes. J. Periodontol., 75:1188-95, 2004.

Prata, C. A.; Lacerda, S. A. \& Brentegani, L. G. Autogenous bone graft associated with enamel matrix proteins in bone repair. Implant Dent., 16:413-20, 2007.

Rummelhart, J. M.; Mellonig, J. T.; Gray, J. L. \& Towle, H. J. A comparison of freeze-dried bone allograft and demineralized freeze-dried bone allograft in human periodontal osseous defects. J. Periodontol., 60:655-63, 1989.

Saito, A.; Hayakawa, H.; Ota, K.; Fujinami, K.; Nikaido, M. \& Makiishi, T. Treatment of periodontal defects with enamel matrix derivative: clinical evaluation at early healing stages. Bull. Tokyo Dent. Coll., 51:85-93, 2010.

Schallhorn, R. G. Present status of osseous grafting procedures. J. Periodontol., 48:570-6, 1977.

Takei, H. H.; Han, T. J.; Carranza, F. A. Jr.; Kenney, E. B. \& Lekovic, V. Flap technique for periodontal bone implants. Papilla preservation technique. J. Periodontol., 56:20410, 1985.

Tonetti, M. S.; Lang, N. P.; Cortellini, P.; Suvan, J. E.; Adriaens, P.; Dubravec, D.; Fonzar, A.; Fourmousis, I.; Mayfield, L.; Rossi, R.; Silvestri, M.; Tiedemann, C.; Topoll, H.; Vangsted, T. \& Wallkamm, B. Enamel matrix proteins in the regenerative therapy of deep intrabony defects. J. Clin. Periodontol., 29:317-25, 2002.
Dirección para correspondencia:

Gustavo Martínez López

Programa de Magister en Ciencias Odontológicas

Especialidad en Periodoncia

Universidad Autónoma de Nuevo León

MÉXICO

Recibido : 12-10-2011

Aceptado: 28-10-2011 